\title{
STEREOLOGICAL ANALYSIS OF SHAPE
}

\author{
Asger Hobolth And Eva B Vedel Jensen \\ Laboratory for Computational Stochastics, Department of Mathematical Sciences, University of Aarhus, Ny \\ Munkegade, DK-8000 Aarhus C, Denmark \\ e-mail: asho@imf.au.dk, eva@imf.au.dk
}

(Accepted May 13, 2002)

\begin{abstract}
This paper concerns the problem of making stereological inference about the shape variability in a population of spatial particles. Under rotational invariance the shape variability can be estimated from central planar sections through the particles. A simple, but flexible, parametric model for rotation invariant spatial particles is suggested. It is shown how the parameters of the model can be estimated from observations on central sections. The corresponding model for planar particles is also discussed in some detail.
\end{abstract}

Keywords: Fourier descriptors, Gaussian process, radius-vector function, shape, spherical harmonics, starshaped objects, stereology.

\section{INTRODUCTION}

The main practical purpose of stereology is to extract quantitative information from microscope images. The traditional parameters estimated from such images relate to number, length, surface area and volume. Most of the stereological techniques for estimating these traditional parameters are valid without specific assumptions about the spatial structure under study. This is the strength of stereology.

In some fields of application, for instance in cancer diagnostics, it is however important to obtain information on additional parameters relating to the organisation of the spatial structure, including location of particles (inhomogeneity), orientation of particles (directional information) and shape variability. In such cases, it seems necessary to adopt a more classical statistical approach and consider specific stochastic modelling of the spatial structure. The present paper is one attempt in this direction. The focus is on parametric shape modelling of a population of spatial particles.

The literature on statistical shape analysis is widely scattered, but books and review papers are available (Bookstein, 1991; Grenander, 1993; Stoyan and Stoyan, 1994; Dryden and Mardia, 1998; Loncaric, 1998; Lele and Richtsmeier, 2000). One of the most important ideas is to represent a random shape as a stochastic deformation of a template shape. Two seminal papers on deformable template models are Grenander et al. (1991) and Grenander and Miller (1994). The specific shape model considered in the present paper is a deformable template model. It is assumed that any particle from the population is bounded and star-shaped relative to a specified reference point belonging to the particle. (The key example concerns cells which are star-shaped relative to their nucleus or nucleolus.) Otherwise, the particles are featureless. Each particle is represented by its so-called radius-vector function, which gives in each spatial direction the distance from the reference point to the boundary of the particle.

The spatial model is a simple, but flexible, three parameter Gaussian model for the radiusvector function, based on a Fourier-Legendre series expansion. One of the model parameters relates to the smoothness of the boundary of a random particle, while the remaining two parameters describe the local and global shape of the particle. Under this model, the distribution of a random particle from the population is invariant under rotation of the particle. The model can be regarded as an extension to threedimensional space of a more well-known Gaussian model for planar particles, based on a traditional Fourier expansion of the planar radius-vector function (Stoyan and Stoyan, 1994; Hobolth et al., 2000). Indeed, Fourier descriptors are commonly used in biological shape analysis (Lestrel, 1997). A version of the spatial model considered in the present paper has originally been described in Miller et al. (1994), and a detailed analysis of the model can be found in Hobolth (2002).

It is possible to develop a stereological analysis of the spatial model where the shape parameters are estimated from central sections through each of a sample of particles. (A central section through a particle is a section through the reference point of the particle.) Such central sections can be sampled in biological cells if physical sections can be replaced by optical microscopic sections. This observation 
technique is well-known in local stereology where methods of estimating more traditional parameters like number, length, surface area and volume from central sections have been developed (Jensen, 1998).

The remaining part of the paper is organized as follows. In Section 2, shape modelling of planar particles is discussed in some detail. This section is meant as an introduction to the concepts involved in the spatial model, presented in Section 3. The stereological analysis of the spatial model is described in Section 4. Section 5 contains an extension of the spatial model to a space-time dynamic model, capable of describing phenomena such as growth.

\section{SHAPE MODELLING OF PLANAR PARTICLES}

Let us consider a planar particle $X$ which is star-shaped relative to a specified reference point, belonging to $X$. The boundary of $X$ can thus be seen in any direction from this point. Without loss of generality, the reference point will be taken to be the origin $O$. The radius-vector function $d(\theta)$ gives the distance from $O$ to the boundary of $X$ along a ray starting at $O$ and with angle $\theta \in[0,2 \pi)$ relative to a fixed axis. Because $X$ is star-shaped we can actually reconstruct $X$ from $d$. In particular, it is possible to express the area and, under regularity conditions, also the boundary length of $X$ in terms of $d$.

Size-invariance is obtained by analysing the normalized radius-vector function

$$
r(\theta)=\frac{d(\theta)}{\frac{1}{2 \pi} \int_{0}^{2 \pi} d(\theta) d \theta},
$$

$\theta \in[0,2 \pi)$. If the particle $X$ is translated, rotated and scaled, then the normalized radius-vector function of the transformed particle with respect to the transformed reference point is the same as the normalized radius-vector function of the original particle $X$ (up to a shift in $\theta$, caused by the rotation). Thus, if we use the more detailed notation $r_{X}(\theta ; O)$ for the normalized radius-vector function of the particle $X$ with respect to the reference point $O$, then

$$
r_{f(X)}(\theta ; f(O))=r_{X}\left(\left(\theta-\theta_{0}\right) \bmod 2 \pi ; O\right),
$$

$\theta \in[0,2 \pi)$, where $f$ is a composition of a rotation around $O$ with angle $\theta_{0} \in[0,2 \pi)$, a translation and a scaling. Below, we will concentrate on the normalized radius-vector function.

We will assume that the particle $X$ is featureless such that its boundary does not contain particular landmarks (like the finger tips of a hand). For shape modelling of featureless objects, it is useful to consider a Fourier series expansion of the normalized radiusvector function

$$
\begin{aligned}
r(\theta)=\frac{b_{0}}{\sqrt{2 \pi}}+\sum_{m=1}^{\infty} b_{m}^{c} & \frac{1}{\sqrt{\pi}} \cos (m \theta) \\
& +\sum_{m=1}^{\infty} b_{m}^{s} \frac{1}{\sqrt{\pi}} \sin (m \theta),
\end{aligned}
$$

$\theta \in[0,2 \pi)$. The Fourier coefficients are

$$
\begin{aligned}
& b_{0}=\int_{0}^{2 \pi} r(\theta) \frac{1}{\sqrt{2 \pi}} d \theta, \\
& b_{m}^{c}=\int_{0}^{2 \pi} r(\theta) \frac{1}{\sqrt{\pi}} \cos (m \theta) d \theta, \quad m \geq 1, \\
& b_{m}^{s}=\int_{0}^{2 \pi} r(\theta) \frac{1}{\sqrt{\pi}} \sin (m \theta) d \theta, \quad m \geq 1 .
\end{aligned}
$$

The first term $b_{0} / \sqrt{2 \pi}$ in the Fourier expansion (Eq. 2) is equal to 1 because of the normalization. Furthermore, it can be seen that $b_{1}^{c}$ and $b_{1}^{s}$ are asymmetry parameters (Hobolth et al., 2000). They are influenced by the position of the reference point $O$. In particular, if $X$ is symmetric with respect to $O$, then $b_{1}^{c}=b_{1}^{s}=0$. Furthermore, if the particle $X$ is a small deformation of a circular disk centered at $O$, then $b_{1}^{c} \approx 0$ and $b_{1}^{s} \approx 0$.

For $m \geq 2$, the geometric interpretation of the Fourier coefficients, $b_{m}^{c}$ and $b_{m}^{s}$, is most easily understood by considering a particle for which all coefficients are zero except for $b_{m}^{c}$ and $b_{m}^{s}$. Such a particle has a normalized radius-vector function of the form

$$
r(\theta)=1+b_{m}^{c} \frac{1}{\sqrt{\pi}} \cos (m \theta)+b_{m}^{s} \frac{1}{\sqrt{\pi}} \sin (m \theta),
$$

$\theta \in[0,2 \pi)$, and exhibits an $m$-fold symmetry in the sense that

$$
r(\theta)=r\left(\theta+\frac{2 \pi}{m}\right)=\cdots=r\left(\theta+\frac{2 \pi(m-1)}{m}\right),
$$

$\theta \in[0,2 \pi / m)$. Note that 2-fold symmetry is the ordinary type of symmetry where the distances from the reference point to the boundary in any pair of opposite directions are the same. We can rewrite Eq. 4 as

$$
r(\theta)=1+\frac{c_{m}}{\sqrt{\pi}} \cos \left(m\left(\theta-\theta_{m}\right)\right),
$$

where $c_{m} \geq 0$ and $\theta_{m} \in[0,2 \pi / m)$ are determined by

$$
\begin{aligned}
& b_{m}^{c}=c_{m} \cos \left(m \theta_{m}\right), \\
& b_{m}^{s}=c_{m} \sin \left(m \theta_{m}\right) .
\end{aligned}
$$


The coefficient $c_{m}$ is the so-called $m$ th phase amplitude while $\theta_{m}$ is the $m$ th phase angle. From Eq. 5 it follows that a particle with normalized radius-vector function of the form (Eq. 4) will typically look like a smoothed regular polygon with $m$ vertices. If $c_{m}$ is small then we will have a circular appearance while for $c_{m}$ large a more distinct shape with $m$ smooth corners is obtained. The interpretation of $\left(c_{m}, \theta_{m}\right)$ is shown in Fig. 1 for $m=3$. A general particle can be regarded as a superposition of such particles and therefore the Fourier coefficients for small $m$ are global shape parameters, while the Fourier coefficients for large $m$ are local shape parameters. Note that because of Eq. 1 and Eq. 5 the phase amplitudes $c_{m}$ are invariant under a rotation of the particle.

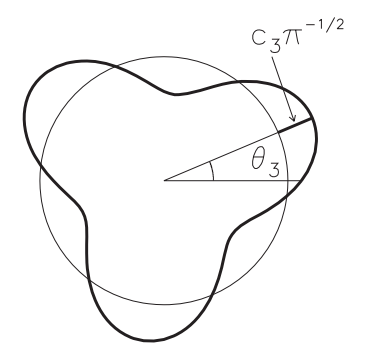

Fig. 1. Interpretation of $\left(c_{3}, \theta_{3}\right)$.

In Fig. 2, the decomposition of a particle into $m$-fold symmetric particles is illustrated. The upper row of Fig. 2 shows the $m$-fold symmetric particles (Eq. 4), $m=2, \ldots, 6$, associated with the particle shown in the lower row. In the lower row of Fig. 2, the original particle is shown together with its reconstruction, using all $m$-fold symmetric particles with $m \leq M, M=2, \ldots, 6$. The normalized radiusvector function of the particle is thereby approximated by the first $M$ terms in the Fourier expansion (Eq. 2). A similar discussion of the geometric interpretation of the Fourier expansion of the tangent-angle function may be found in Zahn and Roskies (1972).

Modelling the shape of a random planar particle from a given population involves specifying the distribution of the Fourier coefficients. Since $b_{1}^{c}$ and $b_{1}^{s}$ have a special interpretation as asymmetry parameters they are not easily modelled. Indeed, the best thing to do is perhaps to consider the parameters separately, but in this paper we desist from such analysis. It is natural to assume that the remaining part of the Fourier expansion

$$
\sum_{m=2}^{\infty} b_{m}^{c} \frac{1}{\sqrt{\pi}} \cos (m \theta)+\sum_{m=2}^{\infty} b_{m}^{s} \frac{1}{\sqrt{\pi}} \sin (m \theta),
$$

$\theta \in[0,2 \pi)$, constitutes a stationary Gaussian process on $[0,2 \pi)$. Then it can be shown that $b_{m}^{c}, b_{m}^{s}, m \geq 2$, are all mutually independent and

$$
b_{m}^{c} \sim b_{m}^{s} \sim N\left(0, \kappa_{m}\right), m \geq 2 .
$$

The variances $\kappa_{m} \geq 0$ determine the covariance function of the normalized radius-vector function

$$
\operatorname{cov}(r(\theta), r(0))=\frac{1}{\pi} \sum_{m=2}^{\infty} \kappa_{m} \cos (m \theta),
$$

$\theta \in[0,2 \pi)$. It is possible to generalize this model to the non-Gaussian case (Hobolth et al., 2000).
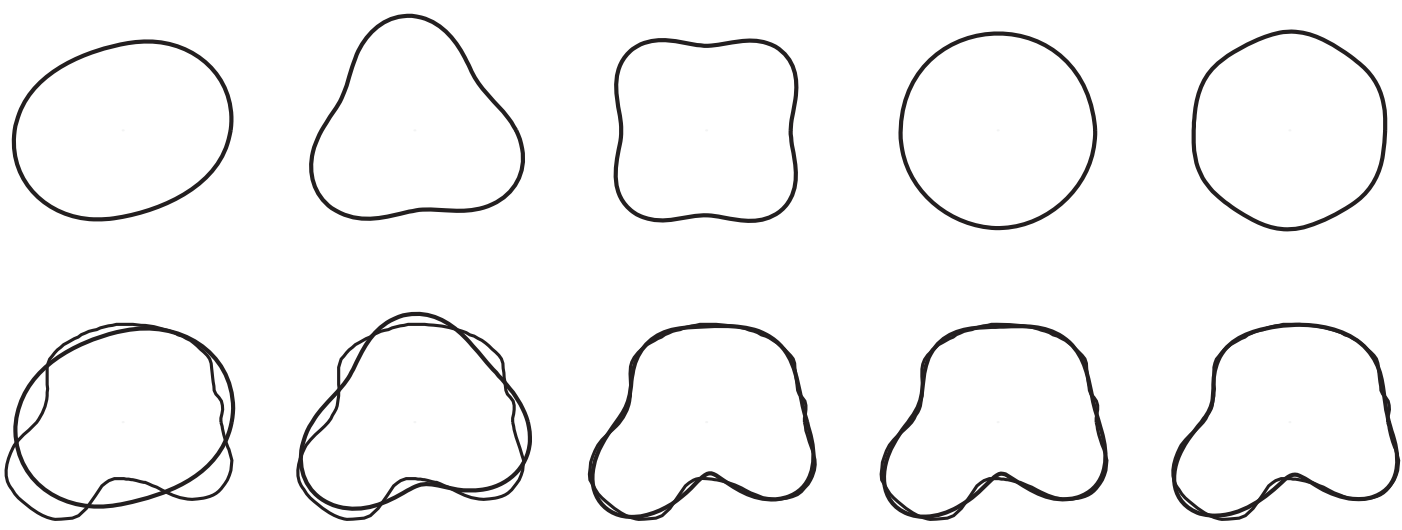

Fig. 2. Decomposition (upper row) of a planar particle in $m$-fold symmetric particles, $m=2, \ldots, 6$, and the corresponding superposition (lower row). 
The variability in particle shape induced by this model is determined by the variances $\kappa_{m}, m \geq 2$. As supported by the discussion of $m-$ fold symmetric particles above, $\kappa_{m}$ will for small $m$ govern the distribution of the global shape and for large $m$ the local shape. In the shape literature, simple parametric regression models for the variances have been suggested

$$
\kappa_{m}=\kappa_{m}(\psi) ;
$$

where $\psi$ is a regression parameter. In particular, the model

$$
\kappa_{m}=\frac{1}{\psi_{1}+\psi_{2}(2 \pi m)^{2}}
$$

has attracted much attention (Kent et al., 2000). In this case, $\psi=\left(\psi_{1}, \psi_{2}\right)$ is a 2 -dimensional vector. In order to ensure that $\kappa_{m} \geq 0, m \geq 2$, the parameters must satisfy

$$
\psi_{2}>0, \psi_{1}>-16 \pi^{2} \psi_{2}
$$

The statistical analysis of such a regression model is straight-forward but should be done with some care because of digitization effects. The regression parameter can be estimated, using the density of the observed Fourier coefficients. Considered as a function of $\psi$, this density is called the likelihood function. Using the first $M$ terms of the Fourier expansion (Eq. 2 ), the likelihood function for a single particle becomes

$$
\begin{aligned}
& L\left(\psi ;\left\{b_{m}^{c}, b_{m}^{s}\right\}_{m=2}^{M}\right)= \\
& \quad=\prod_{m=2}^{M}\left[2 \pi \kappa_{m}(\psi)\right]^{-1} \exp \left(-\frac{\left(b_{m}^{c}\right)^{2}+\left(b_{m}^{s}\right)^{2}}{2 \kappa_{m}(\psi)}\right) \\
& \quad=\prod_{m=2}^{M}\left[2 \pi \kappa_{m}(\psi)\right]^{-1} \exp \left(-\frac{c_{m}^{2}}{2 \kappa_{m}(\psi)}\right)
\end{aligned}
$$

For a sample of particles the individual likelihood functions are multiplied. The parameter estimates maximizes $L$ and are found by standard numerical methods. Note that $L$ only depends on the rotation invariant Fourier coefficients $c_{m}, 2 \leq m \leq M$.

Usually, the normalized radius-vector function of a particle is only known at a discrete set of angles $\theta=0,2 \pi / k, \ldots, 2 \pi(k-1) / k$ and $b_{m}^{c}$ and $b_{m}^{s}$ are approximated using discretized versions of the integrals (Eq. 3) with a large value of $k$. The choice of the cut-off value $M$ is important. If $M$ is too small we loose important shape information whereas if $M$ is too large the results will be influenced by digitization effects because we only know the boundary of the particle with a precision corresponding to the resolution of the digital image.

\section{SHAPE MODELLING OF SPATIAL PARTICLES}

In this section, we show how the methods of the previous section can be generalized to shape modelling of spatial particles. A full and detailed account can be found in Hobolth (2002).

Let us consider a spatial particle $X$ which is starshaped relative to $O$. The spatial radius-vector function of $X$ relative to $O$ is given by

$$
d(\theta ; \phi), \theta \in[0,2 \pi), \phi \in[0, \pi],
$$

where $d(\theta, \phi)$ is the distance from $O$ to the boundary of $X$ in the direction

$$
\omega(\theta, \phi)=(\cos \theta \sin \phi, \sin \theta \sin \phi, \cos \phi) .
$$

Note that $d$ is expressed as a function of the polar coordinates. To remove size-effects we consider the normalized radius-vector function

$$
r(\theta, \phi)=\frac{d(\theta, \phi)}{\frac{1}{4 \pi} \int_{0}^{2 \pi} \int_{0}^{\pi} d(\theta, \phi) \sin \phi d \phi d \theta},
$$

$\theta \in[0,2 \pi), \phi \in[0, \pi$.

Note that $r$ is periodic in $\theta$, but not in $\phi$. Accordingly, the Fourier series expansion in the plane should be replaced by a Fourier-Legendre series expansion

$$
r(\theta, \phi)=1+\sum_{n=1}^{\infty} \sum_{m=-n}^{n} a_{n}^{m} \varphi_{n}^{m}(\theta, \phi),
$$

where

$$
\left\{\varphi_{n}^{m}: n=1,2, \ldots ; m=-n, \ldots, n\right\}
$$

are spherical harmonics (Müller, 1966). The first term in Eq. 8 equals 1 because of the normalization. Each function $\varphi_{n}^{m}$ involves trigonometric functions and Legendre functions. Thus,

$$
\varphi_{n}^{m}(\theta ; \phi)=k_{n}^{|m|} P_{n}^{|m|}(\cos \phi) Q_{m}(\theta),
$$

where

$$
k_{n}^{m}=\sqrt{\frac{2 n+1}{2 \pi} \frac{(n-m) !}{(n+m) !}}
$$

are known constants, $P_{n}^{m}$ are the associated Legendre functions of the first kind (Abramowitz and Stegun, 1965), and

$$
Q_{m}(\theta)= \begin{cases}\cos m \theta & m=-n, \ldots,-1 \\ 1 / \sqrt{2} & m=0, \\ \sin m \theta & m=1, \ldots, n .\end{cases}
$$


As in the planar case, the first coefficients $a_{1}^{-1}, a_{1}^{0}, a_{1}^{1}$ in the expansion (Eq. 8) play a special role. If $O$ is the centroid of $X$ and $X$ is a small deformation of a sphere, then these coefficients are approximately zero. In general, these coefficients carry information about the position of $O$ in $X$ and will not be subject to modelling in this paper. The geometrical interpretation of the remaining coefficients

$$
a_{n}^{m}, n \geq 2, m=-n, \ldots, n,
$$

is similar to the planar case.

In analogy with the planar case, we suggest that the remaining coefficients are modelled by independent Gaussian variables

$$
a_{n}^{m} \sim N\left(0, \lambda_{n}\right), n \geq 2, m=-n, \ldots, n .
$$

It can be shown that the assumption that the variance of $a_{n}^{m}$ does not depend on $m$ ensures that the distribution of the random particle is invariant under rotations (Hobolth, 2002).

Inspired by the physical laws of pressure fields acting on thin membranes, parametric models for the variances $\lambda_{n}$ have been suggested (Miller et al., 1994; Joshi et al., 1997; Grenander and Miller, 1998). In Hobolth (2002), a simplified version of these models is proposed where

$$
\lambda_{n}^{-1}=\alpha+\beta\left(n^{p}-2^{p}\right), \quad n \geq 2,
$$

$p>2, \beta>0, \alpha>0$. The parameter $\alpha$ determines the variances $\lambda_{n}$ for small $n$ which in turn influence the global shape of the random particles produced by the model. The smaller $\alpha$, the larger deviations from spherical shape. The parameter $\beta$ is a local shape parameter since it controls $\lambda_{n}$ for large $n$. A small value of $\beta$ implies irregular particle boundaries on a local scale. Finally, the parameter $p$ determines the smoothness of the boundary of the particle. Simulations of random central sections under the model is shown in Fig. 3 and they support the above mentioned interpretation of the parameters.

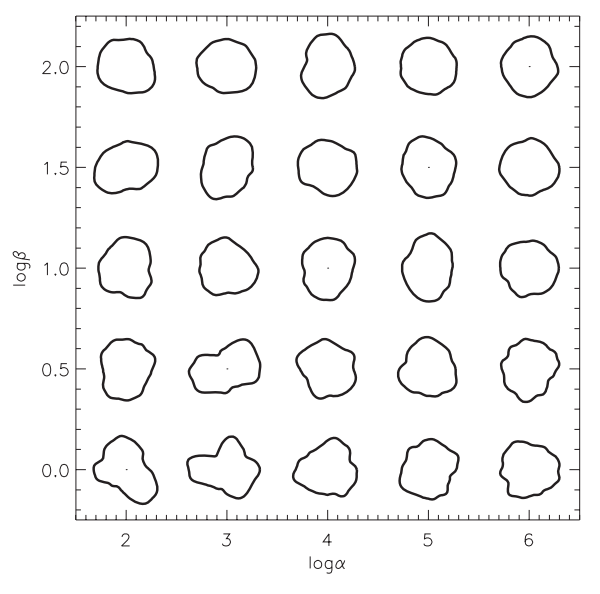

Fig. 3. Central sections through simulated spatial particles under the model (Eqs. 9-10) with $p=4$ and the indicated values of the parameters $\alpha$ and $\beta$.

To understand the shape variability for fixed values of $(\alpha, \beta, p)$ we also simulated random spatial particles from the spatial model, cf. Fig. 4.

\section{STEREOLOGICAL ANALYSIS OF THE SPATIAL MODEL}

The key question is now to develop statistical inference for the model, using measurements on a central section, i.e. a section through $O$. The normalization factor of the spatial radius-vector function

$$
\frac{1}{4 \pi} \int_{0}^{2 \pi} \int_{0}^{\pi} d(\theta ; \phi) \sin \phi d \phi d \theta
$$

cannot be determined from sectional observations but can be estimated using the sectional average.

Under the model (Eqs. 9-10), the distribution of the random particle is invariant under rotations around $O$. Without loss of generality, we can therefore assume that the section is horizontal. The information available is then

$$
\{r(\theta, \pi / 2): \theta \in[0,2 \pi)\}
$$
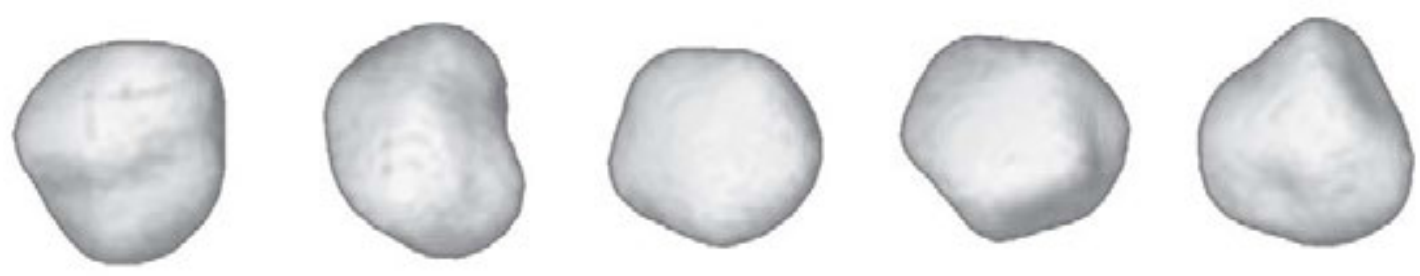

Fig. 4. Random spatial particles with $(\log \alpha, \log \beta, p)=(4,1,4)$. 
Note that if we fix $\phi=\pi / 2$, then Eq. 8 is a Fourier expansion of the form (Eq. 2) with

$$
\begin{aligned}
& b_{m}^{c}=\sqrt{\pi} \sum_{n=m}^{\infty} a_{n}^{-m} k_{n}^{m} P_{n}^{m}(0), \\
& b_{m}^{s}=\sqrt{\pi} \sum_{n=m}^{\infty} a_{n}^{m} k_{n}^{m} P_{n}^{m}(0),
\end{aligned}
$$

$m=1,2, \ldots$. Under the model (Eq. 9), $b_{m}^{c}, b_{m}^{s}, m \geq 2$, are all mutually independent and

$$
b_{m}^{c} \sim b_{m}^{s} \sim N\left(0, \kappa_{m}\right),
$$

where

$$
\kappa_{m}=\pi \sum_{n=m}^{\infty}\left(k_{n}^{m} P_{n}^{m}(0)\right)^{2} \lambda_{n} .
$$

If $\lambda_{n}$ follows the regression model (Eq. 10) for $n \geq 2$, then $\kappa_{m}=\kappa_{m}(\alpha, \beta, p)$ for $m \geq 2$ where

$$
\kappa_{m}(\alpha ; \beta, p)=\pi \sum_{n=m}^{\infty}\left[k_{n}^{m} P_{n}^{m}(0)\right]^{2} /\left[\alpha+\beta\left(n^{p}-2^{p}\right)\right] .
$$

The regression parameters $\psi=(\alpha, \beta, p)$ are estimated by maximizing the likelihood (Eq. 7).

This analysis has been carried out in Hobolth (2002) on a small sample of neurons from the human hippocampus.

\section{DISCUSSION}

Shape modelling of a particle population based on the radius-vector function requires that a reference point belonging to each particle can be selected in some unified way. If particles are cells, then a natural choice of reference point is the nucleus or nucleolus. Instead, the centroid may be used. It should be emphasized that the result of the shape analysis is influenced by this choice of reference point, which may be regarded as a weakness. Also modelling based on an expansion of the radius-vector function (planar or spatial) should be performed with some care since higher order coefficients in the expansions are not determined accurately due to limited resolution.

Alternatives to shape modelling of spatial particle populations by means of the radius-vector function exist (Joshi et al., 1997), but these models do not lend themselves so easily to stereological analysis. If complete spatial observation of the particles is possible, then a generalisation of the pixel-based Gibbs models, described for planar particles in Anastassiou and Sakrison (1981) and Stoyan et al. (2002), is a promising alternative to modelling based on the radius-vector function. Still another approach is shape modelling using elastic deformations, as discussed in a series of papers (Glasbey and Mardia, 1998; 2001).

In the present paper, shape models have been used for describing shape variability in spatial particle populations. Shape models are also useful for modelbased variance estimation (Gual-Arnau and CruzOrive, 2000; Hobolth and Jensen, 2002) and as priors in object recognition (Rue and Syversveen, 1998; Rue and Hurn, 1999).

One of the strengths of the shape modelling approach, taken in the present paper, is that a generalisation to a dynamic set-up is quite easy so that growing particles or particles changing shape over time may be modelled. For a spatial particle, a dynamic set-up can be obtained by letting the random coefficients $a_{n}^{m}$ in the Fourier-Legendre expansion (Eq. 8 ) depend on time. In order to ensure smooth changes in shape, the coefficients $a_{n}^{m}\left(t_{1}\right), a_{n}^{m}\left(t_{2}\right)$ observed at two time points $t_{1}, t_{2}$, respectively, should be correlated. For instance, $\left\{a_{n}^{m}(t): t \geq 0\right\}$ could be modelled as a stationary Gaussian process. Earlier work on dynamic shape models can be found in Cressie and Hulting (1992), Kervrann and Heitz (1999) and Hansen et al. (2002).

\section{REFERENCES}

Anastassiou D, Sakrison DJ (1981). A probability model for simple closed random curves. IEEE Trans Inform Theory 27(3):376-81.

Abramowitz M, Stegun IA (1965). Handbook of Mathematical Tables. New York: Dover Publications.

Bookstein FL (1991). Morphometric Tools for Landmark Data: Geometry and Biology. Cambridge: Cambridge University Press.

Cressie N, Hulting FL (1992). A spatial statistical analysis of tumor growth. Am Stat Assoc 87:272-83.

Dryden IL, Mardia KV (1998). Statistical Shape Analysis. Chichester: Wiley.

Glasbey CA, Mardia KV (1998). A review of image warping methods. J Appl Stat 25: 155-71.

Glasbey CA, Mardia KV (2001). A penalized likelihood approach to image warping. J Roy Stat Soc B 63:465514.

Grenander U, Chow Y, Keenan DM (1991). Hands: A Pattern Theoretic Study of Biological Shapes. Research Notes on Neural Computing. Berlin: Springer.

Grenander U (1993). General Pattern Theory. New York: Oxford University Press. 
Grenander U, Miller M. (1994). Representations of knowledge in complex systems (with discussion). J Roy Stat Soc B 56:549-603.

Grenander U, Miller MI (1998). Computational anatomy: An emerging discipline. Quart Appl Math 5:617-94.

Gual-Arnau X, Cruz-Orive LM (2000). Systematic sampling on the circle and on the sphere. Adv Appl Prob (SGSA) 32:628-47.

Hansen MB, Møller J, Tøgersen FAa (2002). Bayesian contour detection in a time series of ultra-sound images through dynamic deformable template models. Biostat 3:213-28.

Hobolth A (2002). The spherical deformation model. Research Report 18, Laboratory for Computational Stochastics, University of Aarhus, submitted.

Hobolth A, Jensen EBV (2002). A note on design-based versus model-based variance estimation in stereology. Adv Appl Prob (SGSA), in press.

Hobolth A, Pedersen J, Jensen EBV (2003). A continuous parametric shape model. Ann Inst Statist Math, in press.

Hobolth A, Kent JT, Dryden IL (2002). On the relation between edge and vertex modelling in shape analysis. Scand J Statist 29:355-74.

Jensen EBV (1998). Local Stereology. Singapore: World Scientific.

Joshi SC, Miller MI, Grenander U (1997). On the geometry and shape of brain submanifolds. Int J Pattern Recogn Artif Int 11:1317-43.

Kervrann C, Heitz F (1999). Statistical deformable modelbased segmentation of image motion. IEEE Trans Image Process 8(4):583-88.
Kent JT, Dryden IL, Anderson CR (2000). Using circulant symmetry to model featureless objects. Biometrika 87:527-44.

Lele SR, Richtsmeier JT (2000). An Invariant Approach to Statistical Analysis of Shapes. Boca Raton, London: Chapman \& Hall/CRC.

Lestrel PE (ed.) (1997). Fourier Descriptors and their Applications in Biology. New York: Cambridge University Press.

Loncaric S (1998). A survey of shape analysis techniques. Pattern Recogn 31:983-1001.

Miller MI, Joshi S, Maffitt DR, McNally JG, Grenander U (1994). Membranes, mitochondria and amoeba: shape models. In: Mardia K, ed. Advances in Applied Statistics, vol. II. Abingdon, Oxfordshire: Carfax Publishing. 141-63.

Müller C (1966). Spherical Harmonics. Berlin: Springer.

Rue H, Hurn MA (1999). Bayesian object recognition. Biometrika 86:649-60.

Rue H, Syversveen AR (1998). Bayesian object recognition with Baddeley's delta loss. Adv Appl Prob (SGSA) 30:64-84.

Stoyan D, Davtyan A, Turetayev D (2002). Statistics for random domains and particles. In: Mecke K, Stoyan D, eds. Morphology of Condensed Matter. Physics and Geometry of Spatially Complex Systems. Lecture Notes in Physics.

Stoyan D, Stoyan H (1994). Fractals, Random Shapes and Point Fields. Chichester: Wiley.

Zahn CT, Roskies RZ (1972). Fourier descriptors for plane closed curves. IEEE Trans Comput 21:269-81. 